\title{
Correct thermodynamic forces in Tsallis Thermodynamics: connection with Hill Nanothermodynamics
}

\author{
Vladimir García-Morales* , Javier Cervera, Julio Pellicer \\ Departament de Termodinàmica, Universitat de València, C/Dr. Moliner 50, \\ E-46100 Burjassot, Spain
}

\begin{abstract}
The equivalence between Tsallis Thermodynamics and Hill's Nanothermodynamics is established. The correct thermodynamic forces in Tsallis thermodynamics are established. Through this connection we also find a general expression for the entropic index $q$ which we illustrate with two physical examples, allowing in both cases to relate $q$ to the underlying dynamics of the Hamiltonian systems.
\end{abstract}

Key words: Thermodynamics, Statistical Mechanics, Critical point phenomena PACS: 05.70.-a, 05.20.-y, 05.70.Jk

\section{Introduction}

During the last decade there has been growing interest in Tsallis (nonextensive) thermostatistics [1, 2]. This formalism has been successfully applied to a

* Corresponding author. Tel: +34 96354 3119; Fax: +34 963543385

Email address: vladimir.garcia@uv.es (Vladimir García-Morales). 
wide variety of statistical systems at scales ranging from particle and nuclear physics [3] to astrophysics [4] and situations involving long range interactions [5] as well as low dimensional maps and multifractals [6]. Another important success is the straightforward explanation of the occurrence of Levy distributions in nature 7]. The building block of Tsallis thermostatistics, known as Tsallis entropy, was introduced in 1988 [1]

$$
\mathcal{S}^{*}=\frac{\sum_{j}^{\Omega} p_{j}^{q}-1}{1-q} \quad(q \in \Re)
$$

Here $p_{j}$ is the probability of the microstate $j$ and $\mathcal{S}^{*}$ is a generalized entropic measure which reduces to the Gibbs-Shannon one $S=-k \sum_{j}^{\Omega} p_{j} \ln p_{j}$ when the entropic parameter $q$ tends to unity. The main feature of this entropic form is its nonadditivity. Given two subsystems $A$ and $B$ of a composite nonextensive system $A+B$ it can be easily checked [1] that

$$
\mathcal{S}^{*}(A+B)=\mathcal{S}^{*}(A)+\mathcal{S}^{*}(B)+(1-q) \mathcal{S}^{*}(A) \mathcal{S}^{*}(B)
$$

Despite the wide variety of applications that the formalism has proven [2] there are some important points concerning Hamiltonian systems that have not yet been elucidated. Among these, the problem of how can $q$ be calculated for these systems has attracted great attention 8] and is, arguably, the main opened issue of Tsallis thermodynamics (TT) 9]. Another point that has not been clarified is what is the correct form of the thermodynamic forces in TT 10, 11, 12]. It has also been stated that Tsallis entropy cannot have a well defined thermodynamics because of its nonadditivity [10, 13].

Quite interestingly also, there exist already another previous important generalization of traditional thermostatistics which is well grounded physically and which was originally conceived for dealing with nonextensive systems [14]. This 
formalism is Hill's Nanothermodynamics [15] (NT) and was originated in 1962 generalizing the concept of Gibbs' chemical potential to complex nonextensive systems that can vary its entropy by fragmentating in smaller subsystems. NT has been applied succesfully since then to ferromagnetism [16], glassy systems 17] and liquid-vapor interfaces [18, 19]. NT is well grounded physically from its very beginning in contrast with TT which relies in the ad hoc (although beautiful) postulate for the entropy inspired in the multifractal formalism [1]. TT has, however, many practical advantages and allows to deal quite straigthforwardly with complex systems (specially those exhibiting power laws statistically since these can be closely described by means of Tsallis q-exponential distributions). NT could be misunderstood as applicable only to small sizes or small numbers of particles. But stars, clusters and multifractals can be considered also as NT systems (the range of the interactions and/or correlations are of the order of the size of the system or longer) and, in general, all systems in which their equilibrium properties depart from the standard description, which relies in the concept of extensivity [14]. NT is a rigorous statistical foundation of thermodynamic finite-size effects as well as others coming from the complexity of the interactions. It is therefore interesting questioning whether if there is any connection between TT and NT.

In this article we clarify all these points. Specifically: i) We establish the equivalence between TT and NT, ii) this connection allows us then to introduce an adequate form for the thermodynamic forces in TT, overcoming previous difficulties [10] iii) the nonadditivity property is then understood for complex systems thermodynamically and, importantly, iv) we provide an expression for $q$ that can be used to calculate this quantity in Hamiltonian many particle systems relating it to its internal dynamics. 


\section{Formal equations of Tsallis Thermodynamics (TT)}

We present first previous rigorous results of TT and NT to better clarify our approach. Maximization of the Tsallis entropy imposing the constraints $\left\langle X_{\alpha}\right\rangle_{q}=\sum_{j}^{\Omega} p_{j}^{q} X_{\alpha}(j) / \sum_{j}^{\Omega} p_{j}^{q}$ for the biased average $\left\langle X_{\alpha}\right\rangle_{q}$ of an extensive quantity $X_{\alpha}$ leads to recover a Gibbs-like differential equation for the entropy

$$
d \mathcal{S}^{*}=\sum_{\alpha} y_{\alpha} d\left\langle X_{\alpha}\right\rangle_{q}
$$

The rest of the formal equations of TT have been recently established [11]. The Euler equation of TT is

$$
\sum_{\alpha}\left\langle X_{\alpha}\right\rangle_{q} y_{\alpha}=\frac{1}{1-q}\left[1+(1-q) \mathcal{S}^{*}\right] \ln \left[1+(1-q) \mathcal{S}^{*}\right]
$$

and the Gibbs-Duhem equation has the form

$$
\sum_{\alpha}\left\langle X_{\alpha}\right\rangle_{q} d y_{\alpha}=\ln \left[1+(1-q) \mathcal{S}^{*}\right] d \mathcal{S}^{*}
$$

A reasonable condition (local additivity) that variables $\left\langle X_{\alpha}\right\rangle_{q}$ must satisfy and which is used in the derivation of Eqs. (4) and (5) is $d\left\langle X_{\alpha}(\lambda A)\right\rangle_{q} /\left.d \lambda\right|_{\lambda=1}=$ $\left\langle X_{\alpha}(A)\right\rangle_{q}$ for a system $\lambda$ times bigger than another $A$. Note that this by no means imply that $\left\langle X_{\alpha}(\lambda A)\right\rangle_{q}=\lambda\left\langle X_{\alpha}(A)\right\rangle_{q}$ (global additivity) [11], since $\lambda$ is replaced by unity. Global additivity generally holds only when an ensemble of $\lambda$ independent noninteracting systems is considered (see below) but not necessarily upon subdivision of a system into smaller fractions (it is necessary, for example, to provide some energy to separate two droplets $A$ and $B$ from a bigger droplet $A+B$ because of the energetic cost of creating additional interfaces). Tsallis entropy satisfies [1]] $d \mathcal{S}^{*}(\lambda A) /\left.d \lambda\right|_{\lambda=1}=$ $\left[1+(1-q) \mathcal{S}^{*}(A)\right] \ln \left[1+(1-q) \mathcal{S}^{*}(A)\right] /(1-q)$. It is to be noted that, as oc-

curs with their respective counterparts in standard thermodynamics, Eqs. (3) 
to (5) are not independent, i.e. knowing two of them the other one can be trivially deduced. The above general equations and the Legendre transform mechanism allows to determine all thermodynamic quantities of a nonextensive system.

\section{$3 \quad$ Hill Nanothermodynamics (NT)}

Let us now introduce Hill's formalism of NT. It is based in maximization of the Gibbs-Shannon entropy but contains a new entropic thermodynamic potential, the subdivision entropic potential (SEP) $\mathcal{J}$, which couples nonlinearly the natural thermodynamic variables of the system. Introduction of this new potential is made at the ensemble level, in which a set of $\mathcal{N}$ identical, noninteracting small systems is considered [15]. In this case, the set of formal equations are $\mathcal{J} d \mathcal{N}+\sum_{\alpha} y_{\alpha, H} d\left\langle X_{\alpha}\right\rangle_{H, t}=d S_{t}(\mathrm{Gibbs}) ; \mathcal{J N}+\sum_{\alpha} y_{\alpha, H}\left\langle X_{\alpha}\right\rangle_{H, t}=S_{t}(\mathrm{Eu}-$ ler) and $\mathcal{N} d \mathcal{J}+\sum_{\alpha}\left\langle X_{\alpha}\right\rangle_{H, t} d y_{\alpha, H}=0$ (Gibbs-Duhem). Here, subindex $H$ means "Hill's variables" which are the physical (averaged) locally extensive $\left(\left\langle X_{\alpha}\right\rangle_{H, t}\right)$ and intensive $\left(y_{\alpha, H}\right)$ ones. $t$ means "total" and denotes the properties of the whole ensemble. Thus $S_{t}$ is the total entropy for the ensemble of $\mathcal{N}$ systems. We have $S_{t}=\mathcal{N} S$ and $\left\langle X_{\alpha}\right\rangle_{H, t}=\mathcal{N}\left\langle X_{\alpha}\right\rangle_{H}$ in terms of the respective variables for one system (note that here the members of the ensemble do not interact directly although, of course, can exchange heat, volume and particles depending on the ensemble considered, this being itself a formal construction in which total properties are $\mathcal{N}$ times those of one system). The

formal equations of NT become then for one system [15] 


$$
\begin{aligned}
\sum_{\alpha} y_{\alpha, H} d\left\langle X_{\alpha}\right\rangle_{H} & =d S \\
\sum_{\alpha} y_{\alpha, H}\left\langle X_{\alpha}\right\rangle_{H} & =S-\mathcal{J} \\
\sum_{\alpha}\left\langle X_{\alpha}\right\rangle_{H} d y_{\alpha, H} & =-d \mathcal{J}
\end{aligned}
$$

\section{Connection between TT and NT}

Eqs. (6) to (8) are to be compared with Eqs. (3) to (5) respectively. It can be seen that if we make the following connection $S \equiv \mathcal{S}^{*}, y_{\alpha, H} \equiv y_{\alpha},\left\langle X_{\alpha}\right\rangle_{H} \equiv$ $\left\langle X_{\alpha}\right\rangle_{q}$ and

$$
\mathcal{J} \equiv \mathcal{S}^{*}-\left[1+(1-q) \mathcal{S}^{*}\right] \frac{\ln \left[1+(1-q) \mathcal{S}^{*}\right]}{1-q}
$$

the structure of the formal equations of TT and NT is the same. Here the Tsallis entropy $\mathcal{S}^{*}$ is not only the physical one: its property of nonadditivity, see Eq. (2), is also the basis for the SEP $\mathcal{J}$ which is found to be necessary to explain the thermal behavior of small systems (at least). TT describes, thus, the most general thermal equilibrium, the nanothermodynamic equilibrium [20], in which the new potential $\mathcal{J}$ plays a decisive role. $\mathcal{J}$ vanishes for a macroscopic system, for which one has also $q=1$ in Eq.(9), and is a measure of the (thermodynamic) smallness of the system. In making this correspondence it could seem counterintuitive equating an additive entropy $S$ to a nonadditive one $\mathcal{S}^{*}$. However, $S$ is Gibbs-Shannon like because Hill's entropy is written statistically in terms of thermal averages considering a nanosystem in equilibrium with a macroscopic heat bath at a given average temperature. The nonlinear coupling of the intensive variables is accounted for thermodynamically in Eq.(7) by means of the generalized potential $\mathcal{J}$. This is totally equivalent to consider the nanosystem at the same average inverse temperature arising this time not from coupling to a macroscopic heat bath but to a 
neighbouring environment with fluctuating temperature following a chi-square distribution (without need of introducing $\mathcal{J}$ in this case). The latter leads to Tsallis statistics [8, 21], the entropy being nonextensive and the probability distributions depending on the parameter $q$ (that comes from the order of the chi-square (Gamma) distribution considered). Some authors have shown that the Tsallis distribution is the Laplace transform of the chi-square distribution 8, 21]. This is the key point in passing from Boltzmann-Gibbs distributions to Tsallis q-exponential distributions.

Nonadditivity in the entropy (a statistical approach) and introduction of the potential $\mathcal{J}$ (a thermodynamic approach) are only different pathways that lead to the same thermodynamics. As shown below, thermodynamic excess functions (Hill's NT) are linked to entropic nonadditivity (Tsallis TT). It is also to be noted that the ensemble approach followed by Hill to derive the thermodynamics of a small system is equivalent to assume that local additivity of parameters and Eq. (6) hold as well as the following expression for the additivity breaking of the entropy $d S(\lambda A) /\left.d \lambda\right|_{\lambda=1}=S(A)-\mathcal{J}$.

Despite the different statistical behavior both Hill and Tsallis entropies can be made to coincide numerically and the differences of both formalisms concern only the way of averaging. It is worthy insisting on the fact that thermodynamically speaking, however, these differences are irrelevant, since the structure of the formal equations is the same. Both formalisms, TT and NT, preserve the Gibbsian structure of thermodynamics 1, 15]. Fluctuations are also gaussianlike in both formalisms [11, 15]. Furthermore, if the range $0<q<1$ is considered to be the physically meaningful one [11] fluctuations in TT are larger for $q \neq 1$ than in the extensive case [1] which is again consistent with the fact that fluctuations in NT for a system with $\mathcal{J} \neq 0$ are also larger than in the 
$\mathcal{J}=0$ case [15]. Although Tsallis probability distributions are more general than Boltzmann-Gibbs ones, it is important to note that, in NT, $\mathcal{J}$ introduces additional degrees of freedom in the evaluation of the relevant partition function [16] (which in NT is always related to excess quantities besides the traditional thermodynamic ones). Furthermore, and quite importantly, both TT and NT share the property of ensemble non-equivalence for $q \neq 1$ and $\mathcal{J} \neq 0$, respectively.

\section{Correct form for the Lagrange parameters}

It has been questioned the physical meaning of the parameters $y_{\alpha}$, and some authors [11, 12] have concluded previously that the physical quantities of interest are $\hat{y}_{\alpha}=y_{\alpha} /\left[1+(1-q) \mathcal{S}^{*}\right]$. This conclusion, however, is wrong. Parameters $\hat{y}_{\alpha}$ are naturally related to a thermodynamic formalism in which use of Renyi entropy (and not Tsallis entropy) is made. As a function of Tsallis entropy, the Renyi entropy $\widehat{\mathcal{S}}^{*}$ has the form $\widehat{\mathcal{S}}^{*}=\ln \left[1+(1-q) \mathcal{S}^{*}\right] /(1-q)$ [1]. However, while Tsallis entropy is nonextensive and stable [22], Renyi entropy is extensive but thermodynamically unstable [23] and cannot be employed to generalize standard thermodynamics [22]. Parameters $\hat{y}_{\alpha}$ also arise when it is assumed ad hoc global additivity of energy and the maximum Tsallis entropy for two systems brought into contact is considered [12]. This, however, is also incorrect, since energy cannot be globally additive where entropy does not satisfy this property (otherwise, temperature could not be an intensive variable [10]). It is to be noted therefore that neither the correct thermodynamic forces nor the physical meaning of the formal relations were previously established. Through the above correspondence and from Hill's NT, it is now known that the $y_{\alpha}$ 
must be equal for two different systems put in contact at equilibrium. The $y_{\alpha}$ are then the physically meaningful thermodynamic forces. This allows to establish equilibrium TT, which is now free from recent criticisms [10] arising from the use of incorrect thermodynamic forces.

\section{Excess quantities. Meaning of the nonadditivity of Tsallis en- tropy}

Excess thermodynamic quantities $\left\langle X_{\alpha}\right\rangle_{q}^{(x)}$, as considered, for example, in surface physics [15, 24], can be defined as $\left\langle X_{\alpha}(A, B)\right\rangle_{q}^{(x)}=\left\langle X_{\alpha}(A+B)\right\rangle_{q}-$ $\left\langle X_{\alpha}(A)\right\rangle_{q}-\left\langle X_{\alpha}(B)\right\rangle_{q}$ By applying Eq. (3) to the composite system $A+B$ and then to each system $A$ and $B$ separately and using these definitions, we obtain $d\left[(1-q) \mathcal{S}^{*}(A) \mathcal{S}^{*}(B)\right]=\sum_{\alpha} y_{\alpha} d\left\langle X_{\alpha}\right\rangle_{q}^{(x)}$. This clearly leads to quantify the entropy excess in TT as $\mathcal{S}^{*(x)}=(1-q) \mathcal{S}^{*}(A) \mathcal{S}^{*}(B)$ [25]. We see, thus, that TT provides also a specific microscopic statistical model for the thermodynamic excess entropy and this is the very physical meaning of the nonadditivity property. Each independent system $A$ or $B$ is analogous to a bulk phase. Despite their statistical independency, a thermodynamic coupling exists however between both systems. This can be modelled as an interface separating them and contributing with an excess entropy $\mathcal{S}^{*(x)}$ to the properties of the total system $A+B$. A nonextensive system in TT can hence be understood as a twophase-like system at the vicinity of the critical point (since the nonadditivity property holds at all scales which means having always significant interfaces separating clusters of $A$ from clusters of $B$ ). It is interesting that Eq.(9) can be recasted as $\mathcal{J}=\mathcal{S}^{*}-\widehat{\mathcal{S}}^{*}-(1-q) \mathcal{S}^{*} \widehat{\mathcal{S}}^{*}$ which is also similar to the Tsallis entropy scheme of nonadditivity, see Eq.(2). The SEP $\mathcal{J}$ represents the net 
balance between fragmentation or aggregation of the system [15, 16, 17]. This $\mathrm{SEP}$ is hence to be viewed in $\mathrm{TT}$ as a thermodynamic force which can be understood formally in terms of an equilibrium of the actual physical nonextensive system (with physical entropy $\mathcal{S}^{*}$ ) with its completely fragmentated and uncorrelated state in which the system is reduced to a gas of its noninteracting constituents. The gas has entropy $-\widehat{\mathcal{S}}^{*}$. The minus sign comes from the fact that this gas represents disintegration, against the cohesion (the positive Tsallis physical contribution) which preserves the integrity of the nonextensive system. This equilibrium is made through an interface contributing with $-(1-q) \mathcal{S}^{*} \widehat{\mathcal{S}}^{*}$. Note that Renyi entropy $\widehat{\mathcal{S}}^{*}$ is extensive, and hence it describes well this completely fragmentated extensive dilute gas (which is an unstable and supersaturated one) of the physical system.

It can be seen also that $\mathcal{J}$ and $\mathcal{S}^{*}$ are related differentially as $d \mathcal{J}=-(1-$ q) $\widehat{\mathcal{S}}^{*} d \mathcal{S}^{*}$. This equation is somewhat unusual and has no counterpart in standard thermodynamics. If we define $f \equiv d \mathcal{J} / d \mathcal{S}^{*}$ we see that $f(\lambda A)=\lambda f(A)$. This means that the chance to aggregate depends linearly on the size of the system, which is quite reasonable.

\section{General expression for $q$ and examples}

By using our definition of excess entropy, we obtain

$$
1-q=\frac{\mathcal{S}^{*(x)}}{\mathcal{S}^{*}(A) \mathcal{S}^{*}(B)}=\frac{S^{(x)}}{S(A) S(B)}
$$

since $\mathcal{S}^{*}(A) \equiv S(A)$ and $\mathcal{S}^{*}(B) \equiv S(B)$ through our connection while Eq. (6) holds also in NT for the composite system $A+B$ as well as for each separate system $A$ and $B$. In Eq. (10) all the quantities appearing in the 
r.h.s can be always calculated from NT, which means that there is a way to know $q$ from first principles estimations. We now illustrate this assertion by calculating $q$ for nonpolar, nonstructured liquids (as argon) at the vicinity of the critical point. Liquid clusters develop inside the supersaturated vapor phase at all scales. From critical point theory [24] it is well known that the surface tension $\sigma$ scales at the vicinity of the critical point $\left(T_{r} \rightarrow 0\right)$ with the reduced temperature $T_{r} \equiv\left(1-T / T_{c}\right)$, where $T_{c}$ is the critical temperature, as $\sim \sigma_{0} T_{r}^{\mu}$ (with $\mu \approx 1.26$ in three dimensions, and $\sigma_{0}$ being the surface tension amplitude). The correlation length of phase $i(i=A, B), \xi_{i}$, scales as $\sim \xi_{0 i} T_{r}^{-\mu /(D-1)}[24]$ where $D$ is the dimension and $\xi_{0 i}$ the amplitude of the correlation length. In this limit, the bulk entropies can be written in terms of the critical bulk entropic density $s_{c}$ as $S_{i}=s_{c} V_{i} \approx s_{c} \xi_{i}^{D}$, where $V_{i}$ are the volumes occupied by each phase. The excess entropy $S^{(x)}$ is equal to $-a d \sigma / d T$ [15] where $a \approx \xi^{D-1}$ is the surface area. Replacing all these quantities (taking $A$ as the liquid phase) in Eq. (10) and considering $D=3$ we obtain

$$
1-q=\frac{\mu}{4 \pi \omega_{c} s_{c}^{2} \xi_{0 A}^{3} \xi_{0 B}^{3}} T_{r}^{3 \mu-1}
$$

where we have introduced the critical wetting parameter $\omega_{c}=k T_{c} /\left(4 \pi \xi_{0 A}^{2} \sigma_{0}\right) \approx$ 0.78 which is a universal constant for all fluids [19, 26]. We have shown recently that $\omega_{c}$ is related directly to the dynamics of the critical clusters [19]. Concretely, a value $\omega_{c}=0.78$ means that the critical clusters behave universally as a combination of translation and vortex rotational motion 19]. This calculation then relates the entropic parameter $q$ to the dynamical quantity $\omega_{c}$ and the universal exponent $\mu$ that can be obtained separately from renormalization group theory [24]. It is to be noted that although renormalization group theory is an exact approach given the Hamiltonian, real systems exhibit nonideal features due to the complexity of the interactions that can lead to 
departures from the translationally invariant hamiltonians usually considered in these cases. This is particularly dramatic when studying ferromagnetism [16, 20]. In our specific example, classical scaling laws are used only here as an approximation. It is to be noted that far from the critical point (always following the coexistence line) $q$ does not necessarily departs from unity since the bulk entropies do not scale with the correlation lengths. Eq. (11) holds only approximately as the critical point is approached (as assumed from the scaling laws above used). It is to be noted that at the critical point $q=1$. This is consistent with the fact that the two phases in coexistence merge then into a single macroscopic homogeneous one provided that the size of the clusters tend to infinity. This means that, in addressing clusters, the thermodynamic limit is taken at the critical point $(N \rightarrow \infty$ and $V \rightarrow \infty)$ and hence standard thermodynamics should be regained as is the case here. The r.h.s. of this equation is vanishingly small for non-structured simple fluids, as argon, because of the large numerical values for $s_{c}$. For these fluids, then, $q \approx 1$ and this justifies the success of the Gibbs method of the dividing surface within standard thermostatistics in regarding these systems. Departures from this ideal behavior are observed, however, for complex structured liquids as water [24] in which there exists anomalies due to the presence of hydrogen bonding and dipole orientation which drammatically complicates the statistical description. The presence of structuredness reduce the bulk entropy of the liquid and, furthermore, interfacial effects strongly increase (as it is known from the value of the surface tension of water which surprisingly high when compared to simple fluids [24]). It is then to be expected $q$ to depart significantly from unity in liquid water.

There are many other systems as Ag nanoparticles for which surface func- 
tions can be significantly higher than bulk values [27] which would lead, from Eq.(10), to values of $q$ significantly different from unity. Ferromagnetic materials have been also proven to significantly depart from standard thermostatistics [16], and this has been interpreted succesfully in terms of a nanothermodynamic equilibrium [16, 20].

Another example of a formal two-phase-like system is a correlated ionic liquid in the vicinity of a strongly charged macroion [28]. In this situation the behavior of the correlated counterions at the vicinity of the macroion is quite different to those far from the macroion (constituting a Poisson-Boltzmann uncorrelated and disordered liquid) [28, 29]. For weak coupling, the correlated liquid is still a 3D fluid-like one but the rescaled interfacial area available to each molecule is affected by correlations and is approximately given by $2 \Xi$ (where $\Xi$ is the coupling parameter entering in the Hamiltonian) 29]. It is clear, then, that in the weak coupling regime $(0<\Xi<1, q \lesssim 1)$ and from Eq. (10), $1-q \approx c \Xi$, where $c$ is a small constant (since $S^{(x)}$ depends linearly in the interfacial area). This is consistent with the previously fitted curve for $q(\Xi)$ since in the weak coupling limit we have $1-q=1-1 /(1+0.091 \Xi)^{0.68} \approx 0.062 \Xi$ [28].

\section{Concluding remarks}

We have established a connection between TT and NT which provides a sound and unambiguous physical basis for TT. As a bonus, it has allowed us to justify that the $y_{\alpha}$ 's are the correct physical thermodynamic forces (difficulties

pointed out in [10] are now overcomed) and has led us to two expressions that can be used quantitatively to evaluate the degree of nonextensivity in 
Hamiltonian systems. These are Eq. (9), which introduces the connection between both formalisms relating thermodynamic smallness to nonextensivity, and Eq. (11), which allows for an straightforward evaluation of $q$ from NT. A nonextensive system with $q \neq 1$ can now be understood in the thermodynamic limit as one composed of two entangled phases in a critical regime in which the (fractal) interface separating them, besides other finite size effects, contributes significantly to the total entropy at all scales.

\section{Acknowledgements}

We acknowledge financial support from the MCYT (Ministry of Science and Technology of Spain) and the European Funds for Regional Development (FEDER) under project No. MAT2002-00646.

\section{References}

[1] C. Tsallis, J. Stat. Phys., 52 (1988) 479.

[2] See http://tsallis.cat.cbpf.br/biblio.htm for an updated bibliography on the subject.

[3] F. S. Navarra, O. V. Utyuzh, G. Wilk, Z. Wlodarczyk, Phys. Rev. D, 67 (2003) 114002.

[4] D. F. Torres, H. Vucetich, A. Plastino, Phys. Rev. Lett. 79 (1997) 1588.

[5] R. Salazar, R. Toral, Phys. Rev. Lett., 83 (1999) 4233; S. A. Cannas, F. A. Tamarit, Phys. Rev. B, 54 (1996) R12661.

[6] M. L. Lyra, C. Tsallis, Phys. Rev. Lett., 80 (1998) 53; E. P. Borges, C. 
Tsallis, G. F. J. Añaños, P. M. C de Oliveira, Phys. Rev. Lett. 89 (2002)

254103; G. F. J. Añaños, C. Tsallis, Phys. Rev. Lett., 93 (2004) 020601.

[7] C. Tsallis, S. V. F. Levy, A. M. C. Souza, R. Maynard, Phys. Rev. Lett., 75 (1995) 3589; D. Prato and C. Tsallis, Phys. Rev. E, 60 (1999) 2398.

[8] G. Wilk and Z. Wlodarczyk, Phys. Rev. Lett., 84 (2000) 2770 (2000); C. Beck, Phys. Rev. Lett., 87 (2001) 180601.

[9] A. Cho, Science, 297 (2002) 1268.

[10] M. Nauenberg, Phys. Rev. E 67 (2003) 036114; D. H. E. Gross, Physica A 305 (2002) 99.

[11] E. Vives, A. Planes, Phys. Rev. Lett. 88 (2002) 020601.

[12] S. Abe, S. Martinez, F. Pennini, A. Plastino, Phys. Lett. A 281 (2001) 126; R. Toral, Physica A 317 (2003) 209.

[13] S. R. A. Salinas, Introduçao à Física Estatística (Editora da Universidade de Sao Paulo, Sao Paulo, Brasil, 1997).

[14] R. V. Chamberlin, Science, 298 (2002) 1172.

[15] T. L. Hill, J. Chem. Phys. 36 (1962) 3182; T. L. Hill, Thermodynamics of Small Systems (Dover, New York, 1994)

[16] R. V. Chamberlin, Nature 408 (2000) 337.

[17] R. V. Chamberlin, Phys. Rev. Lett. 82 (1999) 2520.

[18] T. L. Hill and R. V. Chamberlin, Proc. Natl. Acad. Sci. USA 95 (1998) 12779 .

[19] V. Garcia-Morales, J. Cervera, J. Pellicer, Phys. Rev. E 67 (2003) 062103.

[20] R. V. Chamberlin, Phys. Lett. A 315 (2003) 313.

[21] G. Wilk, Z. Wlodarczyk, Chaos Soliton Fract. 13 (2002) 581.

[22] S. Abe, Phys. Rev. E 66 (2002) 046134.

[23] B. Lesche, J. Stat. Phys. 27 (1982) 419.

[24] J. S. Rowlinson and B. Widom, Molecular Theory of Capillarity (Claren- 
don Press, Oxford, 1982).

[25] V. Garcia-Morales, unpublished result.

[26] M. E. Fisher, H. Wen, Phys. Rev. Lett. 68 (1992) 3654.

[27] K. K. Nanda, A. Maisels, F. E. Kruis, H. Fissan and S. Stappert, Phys.

Rev. Lett. 91 (2003) 106102.

[28] V. Garcia-Morales, J. Cervera, J. Pellicer, Physica A 339 (2004) 482.

[29] R. R. Netz, Eur. Phys. J. E 5 (2001) 557. 